\title{
Erratum to: Characterization of the spectrum of insecticidal activity of a double-stranded RNA with targeted activity against Western Corn Rootworm (Diabrotica virgifera virgifera LeConte)
}

\author{
Pamela M. Bachman • Renata Bolognesi - William J. Moar • Geoffrey M. Mueller • \\ Mark S. Paradise $\cdot$ Parthasarathy Ramaseshadri · Jianguo Tan · Joshua P. Uffman • \\ JoAnne Warren • B. Elizabeth Wiggins $\cdot$ Steven L. Levine
}

Published online: 18 September 2013

(C) Springer Science+Business Media Dordrecht 2013

\section{Erratum to: Transgenic Res DOI 10.1007/s11248-013-9716-5}

Due to an unfortunate turn of events, an error was introduced in Table 1 of the above-mentioned publication. Two $\mathrm{LC}_{50}$ values in the first two adjacent rows were inadvertently transposed. Furthermore, in the paragraph just above Figure 1, the words 'control' and 'test' were interchanged. Therefore, the sentence must read: "Additionally, there was no significant effect on CPB growth with mean test and control weights of 8.5 \pm 0.2 and $8.2 \pm 0.4 \mathrm{mg}$, respectively $(\mathrm{p}>0.05)$."

The correct table is published on the next page and should be treated as definitive by the reader.

The online version of the original article can be found under doi:10.1007/s11248-013-9716-5.

P. M. Bachman ( $₫)$. W. J. Moar · G. M. Mueller

M. S. Paradise · J. Tan · J. P. Uffman · J. Warren ·

S. L. Levine

Monsanto Company, 800 N Lindbergh Blvd., St. Louis,

MO 63167, USA

e-mail: pamela.m.bachman@monsanto.com

R. Bolognesi · P. Ramaseshadri · B. Elizabeth Wiggins

Monsanto Company, 700 Chesterfield Parkway W,

Chesterfield, MO 63017, USA 
Table 1 Bioassay results from direct feeding studies with DvSnf7 dsRNA using insects from four Orders representing ten insect families

\begin{tabular}{|c|c|c|c|c|c|c|}
\hline Order & Family & Subfamily & Species & $\begin{array}{l}\text { Bioassay } \\
\text { duration } \\
\text { (days) }\end{array}$ & End points & $\begin{array}{l}\mathrm{LC}_{50}^{\mathrm{a}} \text { or no observed } \\
\text { effect concentration } \\
\text { (ng/mL or } \mathrm{g} \text { diet })\end{array}$ \\
\hline \multirow[t]{7}{*}{ Coleoptera } & Chrysomelidae & Galerucinae & D. virgifera virgifera & 12 & Survival & $4.4^{\mathrm{a}}$ \\
\hline & Chrysomelidae & Galerucinae & $\begin{array}{l}\text { D. undecimpunctata } \\
\text { howardi }\end{array}$ & 12 & Survival & $1.2^{\mathrm{a}}$ \\
\hline & Chrysomelidae & Chrysomelinae & L. decemlineata & 12 & Survival, Growth & $5,000^{\mathrm{b}}$ \\
\hline & Tenebrionidae & Tenebrioninae & T. castaneum & 30 & Survival, Growth & $5,000^{\mathrm{b}}$ \\
\hline & Coccinellidae & Coccinellinae & C. maculata & 24 & Survival, Growth, Development & $3,000^{\mathrm{b}}$ \\
\hline & Coccinellidae & Epilachninae & E. varivestis & 28 & Survival, Growth, Development & $3,000^{\mathrm{b}}$ \\
\hline & Carabidae & Harpalinae & P. chalcites & 35 & Survival, Growth, Development & $5,000^{\mathrm{b}}$ \\
\hline Hemiptera & Anthocoridae & Anthocorinae & O. insidiosus & 9 & Survival, Growth, Development & $5,000^{\mathrm{b}}$ \\
\hline \multirow[t]{2}{*}{ Hymenoptera } & Eulophidae & Entedoninae & P. foveolatus & 21 & Survival & $3,000^{\mathrm{b}}$ \\
\hline & Pteromalidae & Pteromalinae & N. vitripennis & 20 & Survival & $5,000^{\mathrm{b}}$ \\
\hline \multirow[t]{4}{*}{ Lepidoptera } & Noctuidae & Noctuinae & S. frugiperda & 8 & Survival, Growth & $500^{\mathrm{b}}$ \\
\hline & & Heliothinae & H. zea & 12 & Survival, Growth & $5,000^{\mathrm{b}}$ \\
\hline & Crambidae & Pyraustinae & O. nubilalis & 12 & Survival, Growth & $5,000^{\mathrm{b}}$ \\
\hline & Bombycidae & Bombycinae & B. mori & 14 & Survival, Growth & $5,000^{\mathrm{b}}$ \\
\hline
\end{tabular}

${ }^{\mathrm{a}} \mathrm{LC}_{50}$ data reported in Bolognesi et al. (2012)

b No observed effect concentration is equal to the maximum concentration tested in these bioassays. Concentrations ranged from 500 to $5000 \mathrm{ng} / \mathrm{mL}$ or ng/g diet and represent 250-2,500 times the maximum expected environmental concentration of DvSnf7 based on greenhouse expression levels 